
\title{
25 Research Square \\ Feasibility of Endovascular Stenting in the Treatment of Carotid Artery Dissection
}

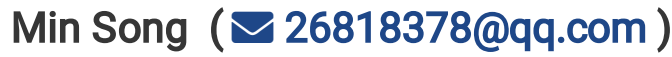

Chongqing Medical University

\section{Xuemin Zhong}

Chongqing Medical University

Gongbo Li

Chongqing Medical University

\section{Yangmei Chen}

Chongqing Medical University

\section{Changqing $\mathrm{Li}$}

Chongqing Medical University

Haiyan Luo

Chongqing Medical University

\section{Research Article}

Keywords: carotid artery dissection, endovascular stenting, ischemic stroke, outcome, stroke in young adults

Posted Date: February 2nd, 2022

DOI: https://doi.org/10.21203/rs.3.rs-1207548/v1

License: (c) (i) This work is licensed under a Creative Commons Attribution 4.0 International License.

Read Full License 


\section{Abstract}

Objective: To clinical curative effect of evaluate the endovascular stenting for the treatment of carotid artery dissection.

Methods: A retrospective analysis of multiple hospital in Chongqing during May 2013 to May 2018 of 53 cases of extracranial NIHSS score of 1 or more clinical data of patients with carotid artery dissection, follow-up the NIHSS and mRS score evaluation of the safety and effectiveness.

Results: The surgical patients with postoperative march mRS compared with preoperative mRS score, score difference was statistically significant; Surgical and non-surgical patient's long-term follow-up mRS score had significant differences.

Conclusion: The results showed that intravascular extracranial carotid stenting treatment is effective and safe in selected patients with acute ischemic stroke, associated with carotid artery dissection.

\section{Introduction}

Carotid artery dissection (CAD) is an important cause of stroke in younger patients and results from a tear in the intimal layer of the artery leading to stenosis or dissecting aneurysm or both. The annual incidence of CAD in epidemiological statistics abroad ranged from 2.6 to $5 / 100,000$ but a common cause of young stroke ${ }^{[1-4]}$.Risk factors reported include genetic susceptibility, minor trauma, migraine, pregnancy and postpartum, past infection or inflammation, connective tissue disease, asphyxia, and hypertension ${ }^{[1]}$. The clinical manifestations of CAD vary, including asymptomatic, mild neurological deficits and symptoms of medullary ischemia leading to respiratory depression ${ }^{[4]}$. Although antithrombotic agents have been used as first-line therapy and have been generally considered sufficient for the treatment of Carotid artery dissections ${ }^{[5]}$. However, stroke patients with large artery occlusion because of carotid artery dissection may have poorer prognosis even if treated with intravenous thrombolysis ${ }^{[6-10]}$. More aggressive and more efficacious treatments may be needed in these patients. Surgical treatment of cervical dissecting artery includes endovascular stenting, endovascular thrombectomy, excision of diseased artery, intravenous vascular replacement, etc ${ }^{[11]}$. Endovascular therapy using stent reconstruction has been utilized as a safe and effective option for certain circumstances ${ }^{[12-16]}$. However, there are no large samples of randomized and controlled trials to evaluate the efficacy and safety of stent implantation. In recent years, some small sample clinical studies have found that endovascular stenting is effective and safe in reducing the recurrence of stroke, and it has become the fundamental method for the treatment of extracranial Carotid artery dissection. In this study,we sought to review our experience and report immediate and long-term outcomes of patients with symptomatic carotid artery dissection from multiple hospitals were treated using surgical or nonsurgical treatments.

\section{Clinical Data And Methods}




\subsection{Clinical data}

\subsubsection{Patient selection}

Clinical data was collected from 53 patients with symptomatic carotid artery dissection between January 2013 and June 2018 at the Second Affiliated Hospital of Chongqing Medical University, Xinqiao Hospital Affiliated to Army Medical University, Chongqing Three Gorges Central Hospital, and Chongqing Fifth People's Hospital. Among them, 41 were male and 12 were female. The ratio of males to females was $3.42: 1$, and the ages ranged from 19 to 67 years. The average age of the operation group was $50.96 \pm 8.3$ years whereas the average age of the non-operation group was $(48.8 \pm 12.3)$ years. All the patients underwent blood biochemical tests. As a result, some patients underwent autoimmune related tests and thyroid function-related examinations. All the patients underwent whole brain digital subtraction angiography before surgery, and some patients performed high-resolution magnetic resonance imaging, computed tomography angiography (CTA), or cervical vascular ultrasound. Carotid artery dissection was diagnosed by cerebral angiography, and the location and nature of the lesion were analyzed. Among the 53 patients who participated in the study, 45 had a single extracranial internal carotid artery dissection, four had bilateral extracranial internal carotid artery dissection, one had intracranial artery dissection, one had middle cerebral artery dissection, and three had vertebral artery dissection. The main manifestations of the lesions were post-dissection stenosis in 50 cases and dissecting aneurysm in three cases. Among them, 24 patients received antithrombotic therapy as decided by the treating physician and could either include anti-platelet therapy or anticoagulation using warfarin with an INR goal of 2-3. but the effect was poor (invalid condition or progress). Endovascular stent angioplasty was performed on the operation group and 29 patients only received anti-platelet therapy or anticoagulation treatment for the nonoperation group. The MRS scores of surgical and non-surgical patients were recorded after three months.

\subsubsection{Inclusion criteria}

a) Symptomatic carotid artery dissection with lumen stenosis or aneurysm formation (NIH Stroke Scale score (> 1 point) confirmed by cerebral angiography (DSA); b) Invalid drug treatment, continued progression of the disease, or imaging examination indicated aggravation of the lesion (the control group did not undergo surgical treatment as a non-parallel control group).

\subsubsection{Exclusion criteria:}

a) Sensitivity to contrast media; b) Intolerance to dual antiplatelet drugs (aspirin and clopidogrel); c) Hematological diseases or severe bleeding tendency; d) Active bleeding three weeks before the operation e) Obvious tortuosity or ripple of diseased vessels leading to difficulty in operation; f) Severe cardiac, pulmonary, and renal diseases; g) Concurrent with other vascular dissections. 


\subsection{Method}

\subsubsection{Antithrombotic regimen}

Patients received dual antiplatelet therapy (aspirin $100 \mathrm{mg}$ and clopidogrel $75 \mathrm{mg}$ ) daily for at least 5 days. Patients who could not receive dual antiplatelet therapy for that period were loaded with oral aspirin $300 \mathrm{mg}$ and clopidogrel $300 \mathrm{mg}$ on the day of the procedure. Dual antiplatelet therapy was continued for at least 3 months after the procedure beyond which only a single antiplatelet agent (aspirin $100 \mathrm{mg}$ or clopidogrel 75 mg daily) was continued.

\subsubsection{Surgical treatment}

Procedures were performed with patients under monitored anaesthesia care. Intravenous heparin was given during the procedure to maintain an activated clotting time between 250 and 300s. A trans-femoral arterial approach was used, and an 8 French sheath was positioned in the common carotid artery. Microcatheter access distal to the lesion was obtained in all cases and angiography was performed to confirm intraluminal position. The use of distal embolic protection was operator dependent and was used when the severity of the stenosis did not preclude passage of the protection device, part of patients was not placed due to the location of the lesion is located in the Carotid siphon or higher. Pre-stent angioplasty was done in selected cases when it was deemed necessary to improve lumen diameter for the safe passage of the stent delivery system. Stent implantation was performed per standard protocols.

\subsubsection{Image analysis}

All images (CTA/MRA/DSA) were reviewed. The DSA images were analyzed to record the characteristics of the dissected vessel for the location, degree of stenosis (defined by the NASCET Criteria), type of stenosis (smooth or irregular), presence or absence of intimal flap, false lumen, presence of thrombus and/or distal emboli and presence and characteristics of dissecting aneurysm ${ }^{[6-8]}$.

\subsubsection{Postoperative management}

Post the operation, ECG, blood oxygen, and blood pressure were monitored for two days to observe the pulsation of the lower limb artery ${ }^{[9-12]}$. The blood pressure was controlled by urapidil or nicardipine for three days after operation and was 20-30\% lower than the baseline blood pressure. Further, the related symptoms of nervous system were observed. The occurrence of any recurrent stroke, TIA, cardiovascular event, or death was recorded.

\subsubsection{Follow-up time}


The average follow-up time was 21.8 months in the operation group and 17.5 months in the nonoperation group. The second average follow-up time was 34.2 months and 25 months in the nonoperation group ${ }^{[6,8,11]}$.

\subsubsection{Clinical outcome analysis}

The preoperative and postoperative mRS scores, long-term follow-up mRS scores, and recurrence rates of stroke (ischemic or hemorrhagic) were recorded in the operation and non-operation groups. The perioperative complications and the success rate of the operation were counted. According to the NIHSS score at admission, the patients were divided into $<4$ points, $>4$ points, and $<10$ points subgroups respectively.

\subsubsection{Statistical analysis}

SPSS20.0 software (IBM Corp, Armonk, NY, USA) was used for statistical analysis. The measurement data were expressed as mean $\pm s d$, and the comparison between groups was performed by a one-way analysis of variance. $P<0.05$ was set for statistically significant.

\section{Results}

\subsection{Immediate outcomes}

Among the 53 cases of CAD, one case was caused by trauma and the rest were spontaneous CAD. The interval from onset to stent implantation was six to 62 days in the operation group, including 15 cases within two weeks and nine cases in the next two weeks. A total of 40 stents were implanted in 24 patients. Angiography showed that the blood supply recovered well during and after the operation. The success rate of the operation was $100 \%$. Four patients had complications, including two patients with transient bradycardia, one with transient chest pain after symptomatic treatment, and no further progress. One patient with middle cerebral artery occlusion (dissection in series) had cerebral hemorrhage at the infarct site the day after the operation, immediately underwent cerebral hematoma removal and severe sequelae remained.

\subsection{Long-term outcomes}

The follow-up time was six months to five years, three patients in the operation group were lost to followup, and two patients in the non-operation group were lost to follow-up. Therefore, the mean follow-up mRS score of 21 patients in the operation group was $1.05( \pm 1.16)$, which was significantly lower from the preoperative mRS score $3.50( \pm 1.18), P=0.03$. Among all cases of $C A D, 17$ patients $(71 \%)$ in the operation group and eight patients (28\%) in the non-operation group had mRS score of less than two points. The proportion of patients in the operation group that basically recovered social function was significantly 
higher than that in the non- operation group,P $=0.007$. During the follow-up, none of the operation and non-operation patients had recurrence of TIA, stroke, or death.

\section{Discussion}

The incidence of stroke caused by the carotid artery dissection is only $2 \%$ of the total number of stroke patients, but it accounts for nearly $5 \%$ to $25 \%$ of young and middle-aged stroke patients younger than 45 years old, which places a heavy burden on the family of the patients and society [ $\left.{ }^{1}\right]$. According to some epidemiological investigations abroad, the average age of the patients is 49.1 years. The average age of the patients in this study was 49.79 years which is consistent with the literature. The baseline characteristics of all patients are shown in Table 1. Due to the low incidence of CAD, there is no large sample randomized controlled trial on CAD.

Currently, more experimental data refers to the surgical treatment of the carotid stenosis. In this study, 38 stents were implanted in 24 surgical patients in the operation group. The top three stents were Wallstent, Protage_self-expanding stent, and Apollo ball expanding stent $\left.{ }^{[-4}\right]$. However, EPD placement may aggravate the vascular intimal injury, resulting in the development of acute thrombosis or dissection. Therefore, EPD is not necessarily used. Balloon dilatation at the lesion site should be considered comprehensively according to the location of the lesion, type of dissection, thrombosis, and other factors. Therefore, balloon dilatation is mainly limited to stent failure or severe stenosis. There were no operativerelated deaths among the 24 patients who underwent CAD surgery in this study. Three patients had transient bradycardia and chest pain, which were alleviated after symptomatic treatment. The success rate of surgery was $100 \%$. This was also consistent with other small sample and single-center studies (see Table 2 for details). One patient with middle cerebral artery occlusion had a cerebral hemorrhage in the infarcted area on the second day after the operation. The imaging results were analyzed considering the large area of cerebral embolism before the operation, which led to the transformation of the cerebral hemorrhage. In the non-operative group, 29 patients underwent DSA, and two patients had femoral artery puncture complications, which improved after symptomatic treatment. Three patients in the operation group and two patients in the non-operation group were unable to reach via the telephone, which resulted in the loss of a follow-up. The follow-up evaluation of patients' mRS indicated that the improvement of $\mathrm{mRS}$ in the operation group was more evident than that in the non-operation group, suggesting that the operation was effective and helpful for patients to recover their social function. Consistent with our research, In a recent systematic review of studies reporting endovascular treatment of carotid dissection that included 31 published reports, the technical success rate of stenting was $99 \%$, and the procedural complication rate was $1.3 \%[17-18]$

All CAD patients were divided into four groups according to the NIHSS, and the improvement in the MRS score of surgical patients with NIHSS scores less than four points was significantly better than that of the non-surgical group; the difference was statistically significant. The improvement of mRS score of surgical patients with NIHSS score greater than four points was significantly better than that of the non-surgical group, and the difference was statistically significant. When the NIHSS score was 4-10 or more than 10 
points, it was observed that there was no significant difference in the improvement of the patients in the operation group and the non-operation group. It was speculated that poor collateral circulation might lead to lower lateral cerebral perfusion in patients with dissecting lesions, resulting in higher NIHSS scores and no significant difference in postoperative recovery and non-operation groups. Patients with lower NIHSS scores had better collateral circulation and better recovery after surgery. It is suggested that patients with NIHSS scores less than 10 have more obvious surgical benefits. No stroke, cerebral infarction, or death occurred during follow-up with Arauz and other small sample size analysis results are consistent[1,19-20], but in a three-month follow-up analysis of 970 CAD patients, the recurrence rates of TIA and strokes were $2.3 \%$ and $1.4 \%$, respectively $\left[{ }^{21}\right]$. In this study, there was no TIA or stroke recurrence in the operation and non-operation groups after follow-up, which may be smaller than the sample size and follow-up time of the study. Short is related.

A study of 38 patients with carotid artery dissection (including 14 patients whose onset time was 8 hours) found that all patients had a good prognosis after the stent implantation ${ }^{[22]} .30$ patients were followed up for 90 days with an mRS score of less than two points. The follow-up of the 14 patients whose onset time was less than 8 hours (acute stage), due to the small sample size of this study and the insufficient sample size for subgroup analysis, was not carried out. Based on previous studies on extracranial carotid artery dissection ${ }^{[22-26]}$ and this study, endovascular stent implantation is safe and effective in the treatment of CAD. However, most of the current studies are data from a small sample size and single-center study. Although this is a multi-center study with a small sample size; large-scale, multicenter, and randomized controlled studies are needed to draw more reliable conclusions.

\section{Declarations}

\section{Acknowledgements}

Thanks to chongqing Municipal Health Commission for support

\section{Authors' contribution}

$\mathrm{XMZ}$ and GBL were responsible for the study conception and design, data collection, first draft of the paper, and final manuscript. XMZ and GBL make the same contribution to the articleChangqing $L i$ and Yangmei Chen were responsible for the first draft of the paper, and final manuscript.Min Song and Haiyang luo was responsible for the conception and design of the study and data analysis and interpretation.All authors read and approved the final manuscript for publication.

\section{Funding}

The project was partly supported by Chongqing health appropriate technology promotion project(2016jstg014) 


\section{Availability of data and materials}

The datasets used and/or analyzed during the current study are available from the corresponding author on reasonable request.

\section{Ethics approval and consent to participate}

Ethical approval for this study was obtained from the Medical and Health Research Ethics Committee of the Second Affiliated Hospital of Chongqing Medical University, chongqing, Chian. Written informed consent was obtained from all the participants. All methods were carried out in accordance with relevant guidelines and regulations.

\section{Consent for publication}

Not applicable.

\section{Competing interests}

The authors declare they have no competing interests.

\section{References}

1. Debette S, Leys D. Cervical-artery dissections: predisposing factors, diagnosis, and outcome. Lancet Neurol. 2009;8:668-678. doi: 10.1016/S1474-4422(09)70084-5

2. Bogousslavsky J, Despland PA, Regli F: Spontaneous carotid dissection with acute stroke. Archives of neurology 44: 137-140, 1987

3. Bogousslavsky J, Pierre P: Ischemic stroke in patients under age 45. Neurol Clin 10: 113- 124, 1992.

4. Leys D, Bandu L, Henon H, Lucas C, Mounier-Vehier F, Rondepierre P, et al.: Clinical outcome in 287 consecutive young adults (15 to 45 years) with ischemic stroke. Neurology 59: 26-33, 2002.

5. Guillon B, Brunereau L, Biousse V, et al. Long-term follow-up of aneurysms developed during extracranial internal carotid artery dissection. Neurology 1999;53:117-22

6. Edgell R, Abou-Chebl A, Yadav J. Endovascular management of spontaneous carotid artery dissection. J Vasc Surg 2005;42:854-60; discussion 860.

7. Fava M, Meneses L, Loyola S, et al. Carotid artery dissection: endovascular treatment. Report of 12 patients. Catheter Cardiovasc Interv 2008;71:694-700.

8. Hassan A, Zacharatos H, Rodriguez G, et al. Long-term clinical and angiographic outcomes in patients with spontaneous cervico-cranial arterial dissections treated with stent placement. $J$ Neuroimaging 2012;22:384-93. 
9. Lavallee P, Mazighi M, Saint-Maurice J, et al. Stent-assisted endovascular thrombolysis versus intravenous thrombolysis in internal carotid artery dissection with tandem internal carotid and middle cerebral artery occlusion. Stroke 2007;38:2270-4.

10. Coldwell D, Novak Z, Ryu R, et al. Treatment of posttraumatic internal carotid arterial pseudoaneurysms with endovascular stents. J Trauma 2000;48: 470-2.

11. Asif Kaiz S,Lazzaro Marc A,Teleb Mohamed S et al. Endovascular reconstruction for progressively worsening carotid artery dissection.[J] .J Neurointerv Surg, 2015, 7: 32-9.

12. Ohta $\mathrm{H}$, Natarajan $\mathrm{S}$, Hauck $\mathrm{E}$, et al. Endovascular stent therapy for extracranial and intracranial carotid artery dissection: single-center experience. J Neurosurg 2011;115:91-100.

13. Hassan $A$, Zacharatos $H$, Souslian $F$, et al. Long-term clinical and angiographic outcomes in patients with cervico-cranial dissections treated with stent placement: a meta-analysis of case series. $\mathrm{J}$ Neurotrauma 2012;29:1342-53.

14. Kadkhodayan Y, Jeck D, Moran C, et al. Angioplasty and stenting in carotid dissection with or without associated pseudoaneurysm. AJNR Am J Neuroradiol 2005;26:2328-35.

15. Muller B, Luther B, Hort W, et al. Surgical treatment of 50 carotid dissections: indications and results. J Vasc Surg 2000;31:980-8.

16. Schievink W, Piepgras D, McCaffrey T, et al. Surgical treatment of extracranial internal carotid artery dissecting aneurysms. Neurosurgery 1994;35:809-15; discussion 815-6.

17. Pham M, Rahme R, Arnaout $O$, et al. Endovascular stenting of extracranial carotid and vertebral artery dissections: a systematic review of the literature. Neurosurgery 2011;68:856-66;discussion 866

18. Donas K, Mayer D, Guber I, et al. Endovascular repair of extracranial carotid artery dissection: current status and level of evidence. J Vasc Interv Radiol 2008;19:1693-8

19. Lee V H, Brown R D, Mandrekar J N, et al. Incidence and outcome of carotid artery dissection A population-based study[J]. Neurology, 2006, 143(3):544-544.

20. Beletsky V, Nadareishvili Z, Lynch J, et al. Cervical arterial dissection: time for a therapeutic trial?[J]. Stroke; a journal of cerebral circulation, 2003, 34(12):2856-60.

21. Arauz A, Hoyos L, Espinoza C, et al. Dissection of cervical arteries: Long-term follow-up study of 130 consecutive cases.[J]. Cerebrovascular Diseases, 2006, 22(2-3):150-154.

22. Castrejón I, Ortiz A M, Garcíavicuña R, et al. Are the C-reactive protein values and erythrocyte sedimentation rate equivalent when estimating the 28-joint disease activity score in rheumatoid arthritis?[J]. Clinical \& Experimental Rheumatology, 2008, 26(5):769.

23. Seth R, Obuchowski A M, Zoarski G H. Endovascular repair of traumatic cervical internal carotid artery injuries: a safe and effective treatment option[J]. Ajnr Am J Neuroradiol, 2013, 34(6):12191226.

24. Asif K S, Lazzaro M A, Teleb M S, et al. Endovascular reconstruction for progressively worsening carotid artery dissection.[J]. Journal of Neurointerventional Surgery, 2015, 7(1):32-9. 
25. Haussen D C, Jadhav A, Jovin T, et al. Endovascular Management vs Intravenous Thrombolysis for Acute Stroke Secondary to Carotid Artery Dissection: Local Experience and Systematic Review[J]. Neurosurgery, 2015, 78(5):709.

26. Marnat G, Mourand I, Eker O, et al. Endovascular Management of Tandem Occlusion Stroke Related to Internal Carotid Artery Dissection Using a Distal to Proximal Approach: Insight from the RECOST Study.[J]. Ajnr Am J Neuroradiol, 2016, 37(7):1281-1288.

\section{Tables}

\section{Table 1}

\section{Baseline Characteristics of the Matched Patients}

\begin{tabular}{|c|c|c|c|}
\hline & $\begin{array}{l}\text { Operation } \\
\text { group }\end{array}$ & $\begin{array}{l}\text { Non- } \\
\text { operation } \\
\text { group }\end{array}$ & $\begin{array}{l}P \\
\text { value }\end{array}$ \\
\hline Case & 24 & 29 & \\
\hline Gender $\rrbracket$ Male/Female】 & $17 / 7$ & $5 / 24$ & 0.302 \\
\hline Age, mean (SD) years $\rrbracket$ & $50.96 \pm 8.37$ & $48.8 \pm 12.3$ & 0.474 \\
\hline Hypertension, n (\%) & $10 \otimes 42 \% \rrbracket$ & $9 \varangle 31 \% \bigotimes$ & 0.422 \\
\hline Diabetes mellitus, n (\%) & $3(13 \%)$ & $3(10 \%)$ & 0.805 \\
\hline Hyperlipidemia, n (\%) & $6(25 \%)$ & $9(23 \%)$ & 0.627 \\
\hline History of smoking, $\mathrm{n}(\%)$ & $7(29 \%)$ & $15(52 \%)$ & 0.097 \\
\hline Alcohol intake n(\%) & $3(16 \%)$ & $12(41 \%)$ & 0.020 \\
\hline $\begin{array}{l}\text { Lesion vessels (left carotid artery/right carotid artery/vertebral } \\
\text { artery/bilateral carotid artery) }\end{array}$ & $9 / 9 / 2 / 4$ & $15 / 13 / 1 / 0$ & \\
\hline \multicolumn{4}{|l|}{ NIHSS scores $\llbracket$ cases $\rrbracket$} \\
\hline$\leq 4$ scores $n$ & 9 & 10 & \\
\hline 4 10scores $n$ & 6 & 12 & \\
\hline$\otimes 10$ scores $n$ & 9 & 7 & \\
\hline mRS scores & $3.50 \pm 1.18$ & $3.48 \pm 1.06$ & 0.955 \\
\hline Follow up $\mathrm{mRS} \leq 2 \mathrm{n}(\%)$ & 17ه70.8\%囚 & $8 \rrbracket 29.6 \% \rrbracket$ & 0.007 \\
\hline
\end{tabular}




\section{Comparison of mRS scores between Operation and non-Operation groups}

\begin{tabular}{|lllc|}
\hline GROUP & $\begin{array}{l}\text { Operation group mRS score } \\
\text { Improvement }\end{array}$ & $\begin{array}{l}\text { Non-Operation group mRS score } \\
\text { Improvement }\end{array}$ & P value \\
\hline $\begin{array}{l}\text { NIHSS scores } \leq 4 \\
\text { Scores }\end{array}$ & 1.71 & 0.33 & 0.009 \\
\hline $\begin{array}{l}\text { NIHSS scores }>4 \\
\leq 10\end{array}$ & 3.57 & 0.91 & 0.006 \\
\hline $\begin{array}{l}\text { NIHSS scores>10 } \\
\text { scores }\end{array}$ & 1.86 & 1.43 & 0.361 \\
\hline
\end{tabular}

*NIHSS score refers to the NIHSS score at admission

*mRS score improvement: mRS score at admission-mRS score at the end of follow-up

\section{Supplementary Files}

This is a list of supplementary files associated with this preprint. Click to download.

- nihss410.xlsx

- nihss10.xlsx

- nihss4.xlsx

- 410.xIsx

- $10 . x \mid s x$

- 4.xIsx 